\title{
APLICAÇÃO DE UM MODELO LINEAR LOCAL NA DETERMINAÇÃO DE ALTURAS ORTOMÉTRICAS REFERIDAS AO SISTEMA GEODÉSICO BRASILEIRO
}

\author{
APPLICATION OF A LOCAL LINEAR MODEL IN THE \\ DETERMINATION OF ORTHOMETRIC HEIGHTS REFERRED \\ TO THE BRAZILIAN GEODETIC SYSTEM
}

APLICACIÓN DE UN MODELO LINEAL LOCAL EN
LA DETERMINACIÓN DE ALTURAS ORTOMÉTRICAS
REFERIDAS AL SISTEMA GEODÉSICO BRASILEÑO
Sílvio Luís Rafaeli Neto - Universidade do Estado de Santa Catarina -
Lages - Santa Catarina - Brasil
silvio.rafaeli@udesc.br
Leonardo Josoé Biffi - Universidade do Estado de Santa Catarina -
Lages - Santa Catarina - Brasil
leonardo.biffi@udesc.br

\begin{abstract}
Resumo
0 uso de sistemas de posicionamento global por satélites (GNSS) possibilita obtenção rápida de alturas elipsoidais (h) relativamente precisas. Contudo, nas aplicações de engenharia, é necessário determinar as correspondentes alturas ortométricas (H) referidas ao geóide ou ao Sistema Geodésico Brasileiro (SGB), cujos referenciais são o campo gravitacional terrestre e a maré média no marégrafo localizado em Imbituba (SC), respectivamente. A determinação de $\mathrm{H}$ em relação à superfície geoidal exige técnicas gravimétricas ou 0 uso de modelos geopotenciais globais ou regionais. Em relação ao SGB aplica-se nivelamento topográfico clássico, partindo-se de marcos da Rede Altimétrica de Alta Precisão (RAAP) até o local de interesse. Em tais métodos, as operações são usualmente laboriosas, de altos custos e despendem muito tempo. Este artigo apresenta a determinação de $\mathrm{H}$ referida ao SGB por meio de um modelo linear de primeira ordem calibrado localmente. A metodologia consistiu em utilizar um conjunto de 17 referências de nível (RNs) da RAAP para calibração do modelo e 7 RNs para sua validação. 0 modelo foi aplicado na avaliação das alturas de dois conjuntos de pontos aleatórios em uma bacia hidrográfica urbana. 0 modelo pode ser aplicado em regiões onde não há transição de zona UTM.
\end{abstract}

Palavras-chave: GPS, Altura Elipsoidal, Bacia Hidrográfica.

\section{Abstract}

The use of Global Positioning Satellite Systems (GNSS) allows quick retrieval of relatively accurate ellipsoidal heights (h). However, in engineering applications, it is necessary to determine the corresponding orthometric heights $(\mathrm{H})$ referred to the geoid or Brazilian Geodetic System (BGS), whose references are the Earth's gravitational field and average tide gauge located in Imbituba (SC), respectively. The determination of $\mathrm{H}$, in relation to geoid surface, requires gravimetric techniques or the use of global or regional geopotential models. The classic topographic leveling is applied to the BGS, starting from the High-Precision Altimetric Network (HPAN) landmarks up to the location of interest. In such methods, the operations are usually labor-intensive, 
of high costs and spend much time. This article presents the determination of $\mathrm{H}$ referred to BGS through a first-order linear model that was calibrated locally. The methodology consisted in using a set of 17 references level (RL) of HPAN for the model calibration and 7 RL for its validation. The model application was in heights evaluation of two sets of random points, in an urban watershed. It is possible to apply the model in regions with no transition of UTM Zone.

Keywords: GPS, Ellisoidal Height, Watershed.

\section{Resumen}

El uso de Sistemas Satelitales de Posicionamiento Global (GNSS) posibilita obtención rápida de alturas elipsoidales (h) con una relativa precisión. Sin embargo, en las aplicaciones de ingeniería, es necesario determinar las correspondientes alturas ortométricas $(\mathrm{H})$ referidas al geoide o Sistema Geodésico Brasileño (SGB), cuyas referenciales son el campo gravitacional de la Tierra y la marea media en el mareógrafo ubicado en Imbituba (SC), respectivamente. La determinación de $\mathrm{H}$ en relación a la superficie del geoide, requiere técnicas gravimétricas o el uso de modelos geopotenciales regionales o globales. En relación al SGB se aplica la nivelación topográfica clásica, partiéndose de marcos de la Red Altimétrica de Alta Precisión (RAAP) hasta el lugar de interés. En tales métodos, las operaciones son intensivas, de alto costo y, generalmente, demoran mucho tiempo. Este artículo presenta la determinación de H referida al SGB mediante un modelo lineal de primer orden calibrado localmente. La metodología consistió en la utilización de un conjunto de 17 puntos de referencias de nivel (RNs) de la RAAP para la calibración del modelo y 7 RNs para su validación. El modelo fue aplicado en la evaluación de las alturas de dos conjuntos de puntos aleatorios en una cuenca urbana. El modelo puede ser aplicado en regiones donde no hay transición de zona UTM.

Palabras clave: GPS, Altura Elipsoidal, Cuenca.

\section{Introdução}

O posicionamento por satélites artificiais (GNSS - Global Navigation Satellite Systems) tem possibilitado a obtenção rápida e relativamente precisa da posição horizontal (coordenadas geocêntricas X, Y, Z, geodésicas $\phi, \lambda$ ou cartográficas $E, N$ ) e vertical referida ao elipsóide (altura geométrica ou elipsoidal h). A acurácia fundamentalmente dependente do tempo de rastreio, da qualidade do sinal rastreado e da qualidade das coordenadas da base, quando aplicadas técnicas diferenciais (Arana, 2000; Featherstone et al., 1998). No SGB, estas alturas são referenciadas à superfície do Elipsóide do Sistema Geodésico de Referência de 1980 (Geodetic Reference System 1980 - GRS80).

Apesar da relativa facilidade com que as técnicas GNSS podem ser empregadas no posicionamento horizontal preciso, o mesmo não é possível afirmar quando se trata de posicionamento vertical, especialmente devido a particulares inerentes à superfície utilizada como referencial ou datum. A altura ortométrica $(\mathrm{H})$ de um ponto na superfície topográfica, referida ao geóide, pode ser obtida pela equação $1^{1}$, na qual $h_{i}$ é a altura geométrica ou elipsoidal e $\mathrm{N}$ a distância que separa estas duas superfícies ao longo 
da linha vertical do ponto no terreno, denominada de ondulação geoidal ou altura geoidal:

$$
H_{i} \approx h_{i}-N_{i}
$$

Por esta técnica, a determinação de $\mathrm{N}$ pode ser feita com modelos geopotenciais globais como, por exemplo, o EGM2008 (Pavlis et al., 2012) ou regionais, como o MAPGEO2010 (Matos et al., 2012) a partir das coordenadas geodésicas do ponto.

Embora H obtida por esta técnica incorpore o rigor necessário associado ao campo gravitacional terrestre, muitas bases cartográficas existentes no Brasil estão vinculadas a RNs pertencentes à RAAP. De acordo com IBGE (2013), grande parte destas alturas refere-se ao nível médio do mar, medido entre 1949 e 1957, no marégrafo do Porto de Imbituba (SC). Apesar de suas alturas não estarem referidas ao geóide local, as RNs da RAAP fazem parte da configuração altimétrica oficialmente estabelecida para o Sistema Geodésico Brasileiro (SGB) e têm servido a projetos de engenharia e a mapeamentos em geral. Assim, as RNs da RAAP são bases altimétricas a partir das quais suas alturas podem ser propagadas até a região do projeto, através de nivelamento geométrico composto. Contudo, está-se sujeito à sua disponibilidade, despende-se tempo e é operacionalmente oneroso.

Um método alternativo aos nivelamentos está na utilização de modelos locais para estimativa das alturas ortométricas, a partir das suas respectivas alturas elipsoidais obtidas por posicionamento GNSS. Este trabalho teve por objetivo calibrar, validar e aplicar um modelo linear na determinação das alturas ortométricas de pontos localizados na superfície topográfica. Optou-se por um modelo de primeira ordem, tendo em vista sua simplicidade e facilidade em obter as variáveis de entrada. O modelo foi calibrado e validado para um campo de cerca de $50 \mathrm{~km}$ e aplicado na avaliação de uma base cartográfica digital obtida por fotogrametria na escala 1:2000, abrangendo uma bacia hidrográfica urbana de $30 \mathrm{~km}^{2}$.

Modelo linear para determinação de $\mathrm{H}$

Modelos lineares para determinação de $\mathrm{H}$ têm sido propostos na literatura (Abdullah, 1997; Zhong, 1997; Khalid e Som, 2011), nos quais as 
variáveis de entrada comumente são as coordenadas horizontais e a altura elipsoidal. Uma variação desta abordagem é utilizar como coordenadas de entrada as coordenadas projetivas UTM. O modelo linear apresentado na equação (2) fornece a altura ortométrica em quaisquer pontos " $x$ ” sobre a superfície terrestre em função de $\mathrm{h}_{\mathrm{GNSS}}$ (altura elipsoidal obtidas por posicionamento GNSS) e das suas coordenadas projetivas UTM (E, N):

$$
\mathrm{H}_{\mathrm{x}}=\mathrm{a}_{1}+\mathrm{a}_{2} \mathrm{~h}_{\mathrm{GNSS}}+\mathrm{a}_{3} \mathrm{E}_{\mathrm{GNSS}}+\mathrm{a}_{4} \mathrm{~N}_{\mathrm{GNSS}}
$$

Ou simplesmente,

$$
\mathbf{H}_{\mathrm{X}}=\mathbf{a}^{\mathrm{T}} \mathbf{X}
$$

Onde:

$\mathbf{H}_{\mathrm{x}}$ : vetor das alturas ortométricas dos pontos topográficos "x" na região geográfica em estudo;

$\mathbf{a}^{\mathrm{T}}$ : vetor com os parâmetros adimensionais ajustados $\left[\mathbf{a}^{\mathrm{T}}=\left(\mathrm{a}^{1} \mathrm{a}^{2}\right.\right.$ $\left.\left.a^{3} a^{4}\right)\right]$; $\left.\mathrm{N}_{\text {GNSS }}\right)$.

$\mathbf{X}$ : vetor com as variáveis independentes $(\mathrm{m})\left[\mathbf{X}^{\mathrm{T}}=\left(\begin{array}{lll}1 & \mathrm{~h}_{\mathrm{GNSS}} & \mathrm{E}_{\mathrm{GNSS}}\end{array}\right.\right.$

Os parâmetros do vetor $\mathbf{a}^{\mathrm{T}}$ são obtidos mediante ajustamento (regressão linear múltipla) e h, E e N são determinados por posicionamento GNSS sobre as RNs da RAAP.

Para a calibração, as variáveis de saída são as alturas ortométricas da RAAP, fornecidas pelo IBGE:

$$
\mathbf{H}_{\text {RAAP }}=\mathbf{a}^{\mathrm{T}} \mathbf{X}
$$

O nível de confiança desta medida está relacionado com o grau de confiança das coordenadas obtidas pelo posicionamento GNSS.

Material e métodos

\section{Worflow para obtenção do modelo}

A metodologia consistiu em calibrar e validar o modelo e aplicá-lo na avaliação das alturas digitais da base cartográfica urbana de Lages (SC). A calibração foi realizada sobre 17 RNs e a validação sobre 7 RNs, sendo estas localizadas a cerca de $50 \mathrm{~km}$ de distância das primeiras. O método 
da regressão linear múltipla foi empregado para calibração do modelo e a técnica de validação cruzada (Fotopoulos, 2003) para sua validação. A análise da qualidade dos resultados obtidos nestas etapas foi feita sobre os resíduos, ou seja, sobre as diferenças entre o valor observado e o valor estimado pelo modelo. A Figura 1 apresenta o worflow para obtenção do modelo linear local.
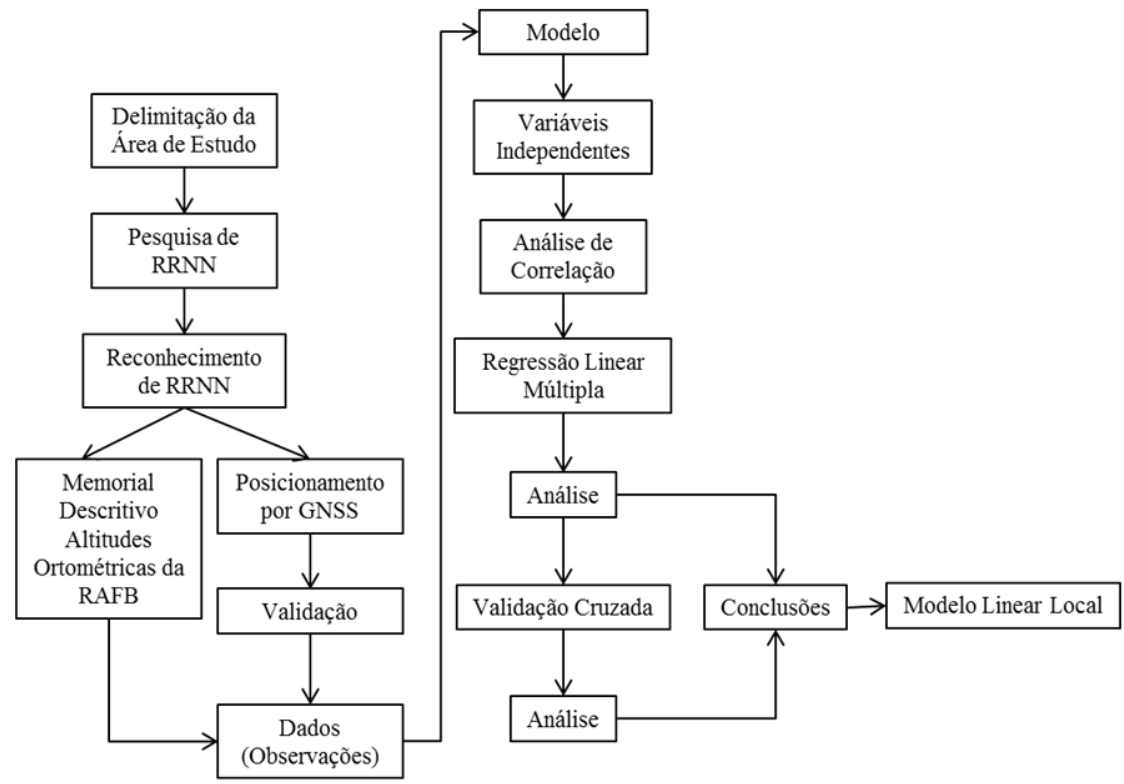

Figura 1 - Wokflow para obtenção do modelo linear local

\section{Caracterização da área de estudo}

O estudo foi conduzido na região de Lages, Planalto Serrano do Estado de Santa Catarina, situada nas coordenadas $-27^{\circ} 49^{\prime}$ de latitude e $-50^{\circ} 20^{\prime}$ de longitude, com cerca de $920 \mathrm{~m}$ de altura média. O clima da região é do tipo $\mathrm{Cfb}$, do tipo mesotérmico, úmido com verão fresco. Nesta região, predominam cambissolos, glei húmicos e litossolos.

A área de estudo foi delimitada pelo divisor de águas da bacia hidrográfica do Rio Carahá, em Lages (Figura 2). O interesse por esta área se justifica por ser uma bacia essencialmente urbana, na qual há um histórico 
de eventos de inundações frequentes nos últimos 20 anos. Grande parte dos $30,2 \mathrm{~km}^{2}$ de sua área territorial está ocupada por edificações ou arruamentos que interferem no escoamento superficial direto oriundo da precipitação. Especificamente, o estudo do geóide nessa área dará suporte à modelagem de áreas de inundações a partir de modelos hidrológicos e hidrodinâmicos.

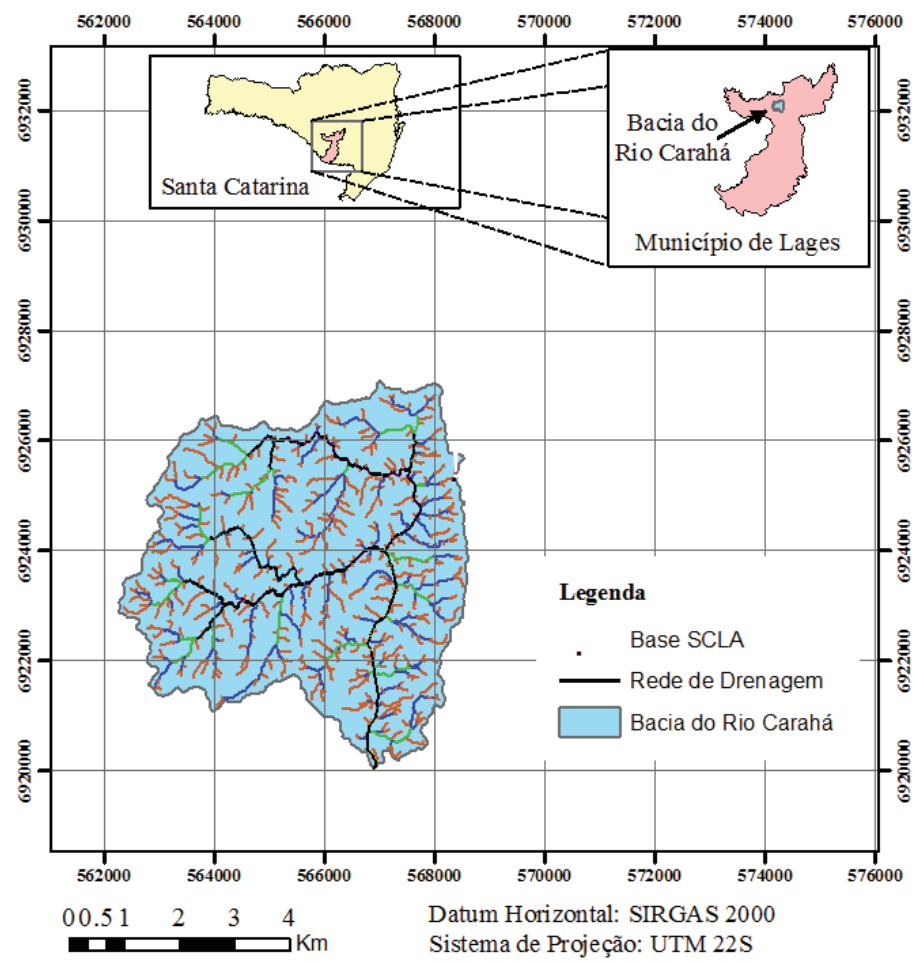

Figura 2 - Bacia hidrográfica do rio Carahá em Lages, Planalto Serrano de Santa

\section{Disponibilidade de RNs na área de estudo}

O Planalto Serrano de Santa Catarina conta com uma rede de referências de níveis pertencentes à RAAP, distribuídas ao longo de rodovias e nas cidades. As alturas ortométricas oficiais dessas RNs, vinculadas ao SGB, podem ser obtidas nos seus respectivos memoriais descritivos disponibilizados no site do IBGE. 
Campanhas de reconhecimento dessas RNs foram realizadas no ano de 2010 com objetivo de localizá-las em campo. Até aquela data, a última visita oficial realizado in loco pelo IBGE nas RNs havia ocorrido em 2006. As alturas registradas nos memoriais utilizados neste trabalho refletem o resultado do ajustamento da rede realizado em julho de 2011 pelo IBGE. Do conjunto total de RNs selecionadas foi possível encontrar 17 (Figura 3).

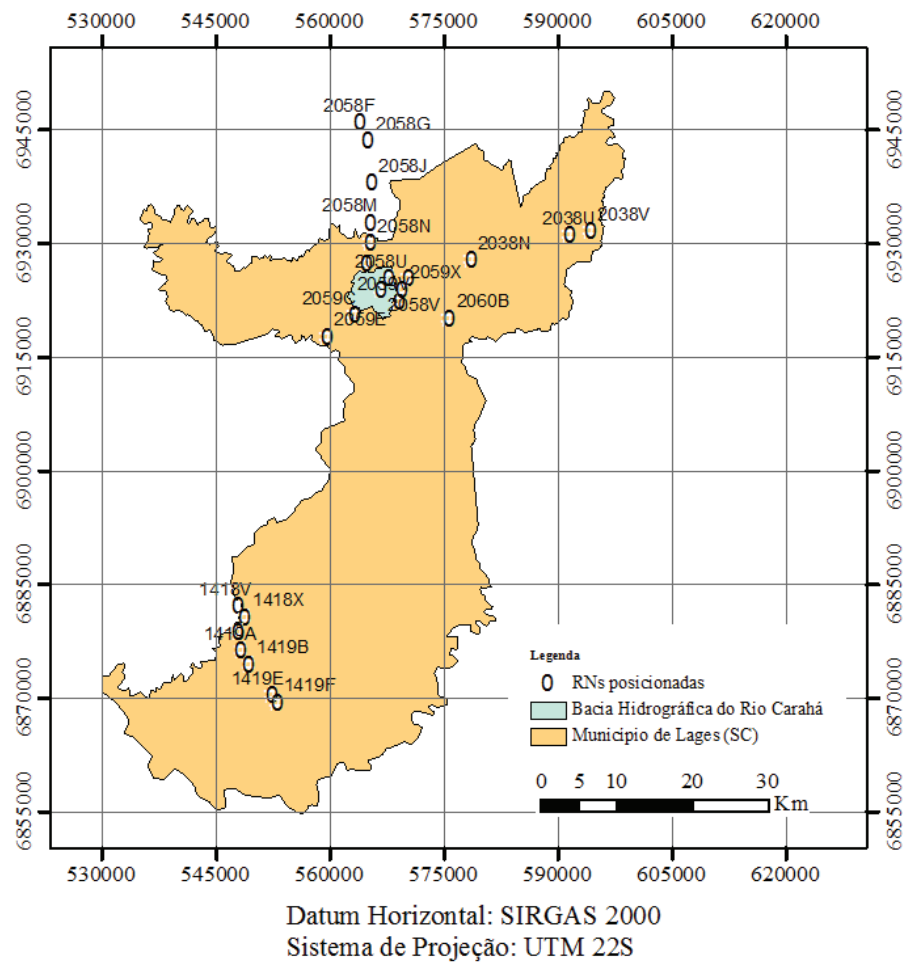

Figura 3 - RNs da RAAP posicionadas por GNSS

\section{Posicionamento das RNs}

As RNs encontradas foram posicionadas por GNSS no modo relativo estático em oito campanhas de rastreio, nas quais se utilizou de um receptor de dupla frequência, marca TOPCON, modelo GR-3, habilitado para rastreio das constelações GPS e GLONASS. O tempo de rastreio foi estabelecido de 
30 a 45 minutos, com taxa de aquisição de cinco segundos (Arana, 2000; IBGE, 2011). O pós-processamento dos dados foi realizado no programa GNSS Solutions, adotando os seguintes parâmetros de projeto: sistema SIRGAS2000, precisão horizontal de $0,020 \mathrm{~m}+1 \mathrm{ppm}$, precisão vertical de 0,040 $\mathrm{m}+2$ ppm. Todas as sessões foram processadas pelo método das duplas diferenças com efemérides, relógios e grelhas ionosféricas precisas, quando disponíveis.

Estabeleceu-se um limiar de aceitação do erro padrão das alturas elipsoidais pós-processadas em $\pm 40 \mathrm{~mm}$, sendo este o desvio-padrão das RNs da RAAP processadas em junho de 2011.

\section{Análise dos resíduos}

As análises estatísticas foram realizadas em dois níveis (Figura 4): a) análise da correlação entre as variáveis independentes utilizadas para ajustar o modelo, b) análise do modelo quanto ao ajuste e à validação.

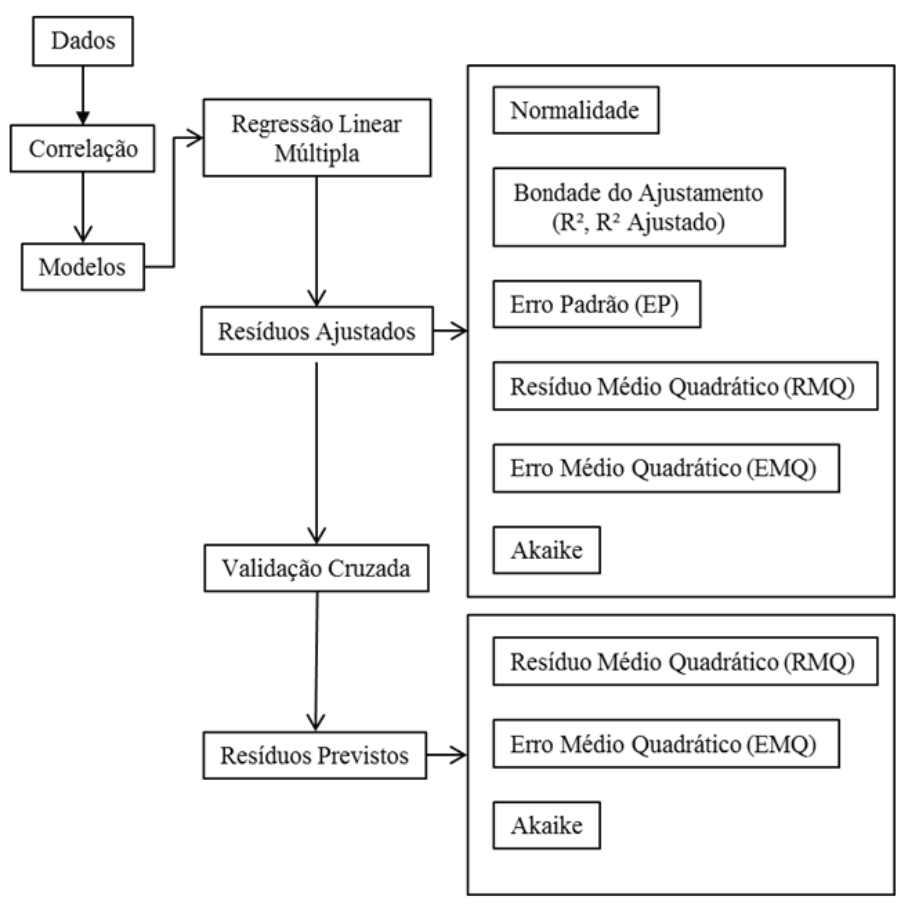

Figura 4 - Metodologia de análise dos resíduos 
Fotopoulos (2003) aponta as metodologias usuais para análise da qualidade da estimativa de um modelo. Na abordagem empírica clássica o modelo é avaliado pela análise dos resíduos (v), confrontando os valores estimados pelo modelo com os valores esperados ou de referência. Este método seria válido para testar a precisão do modelo, mas não sua acurácia, ou seja, sua capacidade de previsão.

No método de validação cruzada, o modelo é ajustado sobre um conjunto de RNs e sua capacidade de previsão é testada em um segundo conjunto, não utilizado no ajustamento. Os resultados dependeriam da acurácia deste último, sendo preferível utilizar tantos dados quanto possível (Fotopoulos, 2003).

No processo de modelagem, é desejável que as variáveis utilizadas no ajuste do modelo, isto é, as variáveis independentes, sejam não correlacionadas entre si, ou seja, a parcela de contribuição de cada uma delas $\left(\mathbf{a}^{\mathrm{T}}\right)$ na estimativa da variável dependente Y não deve incorporar correlação com as demais. O método empregado neste trabalho foi o da Correlação Linear Múltipla realizada em planilha eletrônica.

Para fins de ajustamento do modelo, foram utilizadas coordenadas tridimensionais $\mathrm{E}_{\mathrm{GNSS}}, \mathrm{N}_{\mathrm{GNSS}}, \mathrm{h}_{\mathrm{GNSS}}$ das RNs do grupo “20xxx" como variáveis independentes e a respectiva altura ortométrica $\left(\mathrm{H}_{\mathrm{RAAP}}\right)$ como variável dependente (ou resposta) Y.

O ajustamento compreendeu a estimativa das parcelas de contribuição de cada coeficiente ou parâmetro na estimativa da variável Y, representadas pelo vetor aT. A análise foi realizada através de teste de significância dos coeficientes do modelo e sobre parâmetros indicadores de qualidade.

A validação dos coeficientes ajustados foi realizada pela análise de suas significâncias estatísticas pelos seguintes testes: Análise de Variância (ANOVA); teste $t$ de Student, no qual $t_{\text {calc }}=\mathrm{a} / \mathrm{EP}$, sendo "a" o coeficiente estimado e EP o erro padrão deste mesmo coeficiente; probabilidade de significância dos coeficientes de regressão, ou seja, o p-valor e o intervalo de confiança construído para cada um dos coeficientes estimados.

\section{Avaliação do modelo}

A análise de resíduos (v) é o método estatístico usualmente empregado quando se deseja comparar o desempenho de um grupo ou família 
de modelos (Braga, 2005). Resíduo é definido como a discrepância ou diferença entre o valor de uma variável estimado ou previsto por um modelo e o respectivo valor observado ou medido (Equação 5).

$$
\mathrm{v}_{\mathrm{i}}=V_{o b s}-V_{\text {est }}
$$

Na qual $\mathrm{V}_{\text {obs }}$ é o valor observado ou medido e $\mathrm{V}_{\text {est }}$ o valor estimado ou previsto pelo modelo. Os valores utilizados como variáveis observadas $\left(\mathrm{V}_{\text {obs }}\right)$ foram as alturas ortométricas $\left(\mathrm{H}_{\mathrm{RAAP}}\right)$ das $\mathrm{RNs}$.

Os resíduos produzidos pela estimativa (ou do ajustamento) foram obtidos aplicando-se o modelo nas estimativas das respectivas alturas ortométricas das 17 RNs utilizadas para calibrar o modelo, subtraídas das alturas ortométricas oficiais obtidas do IBGE (todas em metros e ordem de milímetros).

Os resíduos obtidos na validação foram produzidos de forma análoga, porém sobre as 7 RNs utilizadas na validação cruzada. Os valores observados (variável dependente $\mathrm{Y}$ ) do modelo foram as alturas ortométricas $\left(\mathrm{H}_{\mathrm{RAAP}}\right)$ das RNs. Os parâmetros estatísticos utilizados para avaliação dos resíduos (ajustados e/ou da previsão) do modelo foram os seguintes:

1. Condição de normalidade dos resíduos: a normalidade dos resíduos comumente é uma condição para a aplicação da regressão linear múltipla. Dentre os métodos de avaliação da normalidade, o teste de Shapiro-Wilk se destaca por ser aplicável em situações em que o número de observações é pequeno (no caso presente $\mathrm{n}=17)$ e não necessita de agrupamento dos dados. Na avaliação de modelos, o nível de significância de $5 \%(\alpha=0,05)$ seria suficiente para as conclusões (Braga, 2005).

2. $\mathrm{R}^{2}$ : o $\mathrm{R}^{2}$ deve ser analisado juntamente com a estatística $\mathrm{R}^{2}$ Ajustado porque, em casos onde há pouca redundância nos dados (poucos graus de liberdade), pode ocorrer que se obtenha um valor alto para a estatística $\mathrm{R}^{2}$, independentemente da qualidade da calibração (Fotopoulos, 2003).

3. $\mathrm{R}^{2}$ Ajustado: a estatística $\mathrm{R}^{2}$ Ajustado é calculada pelo $\mathrm{R}^{2}$ dividido pelas quantidades (n-p) e (n-1) onde $n$ é o número de observações e po número de parâmetros. Quanto mais próximo $\mathrm{R}^{2}$ Ajustado estiver de $\mathrm{R}^{2}$, mais confiável é o valor de $\mathrm{R}^{2}$ e também os parâmetros calibrados (Montgomery, 2009). Modelos 
com maior número de parâmetros $(p)$ tenderiam a apresentar maiores discrepâncias entre $\mathrm{R}^{2}$ Ajustado e $\mathrm{R}^{2}$.

$$
\mathrm{R}_{\alpha}^{2}=1-\frac{(\mathrm{n}-1)}{(\mathrm{n}-\mathrm{p})}\left(\mathrm{R}^{2}\right)
$$

4. Erro padrão (EP): o erro padrão expressa o desvio médio dos valores observados em relação à curva ajustada.

5. Resíduo médio quadrático (RMQ): informa o resíduo médio da estimativa, sem considerar o número de parâmetros do modelo.

$$
\operatorname{RMQ}=\sqrt{\frac{\mathbf{v}^{\mathrm{T}} \mathbf{v}}{\mathrm{n}}}
$$

6. Erro médio quadrático (EMQ): é um estimador que também considera o número de parâmetros de um modelo, podendo também ser um indicador para escolha de modelos candidatos.

$$
\mathrm{EMQ}=\frac{\mathbf{v}^{\mathrm{T}} \mathbf{v}}{\mathrm{n}-\mathrm{p}}
$$

A equação 8 é uma adaptação de Braga (2005), onde $V$ é o vetor dos resíduos, n é o número de observações e p o número de parâmetros.

7. Erro de predição de Akaike (adaptado de Braga, 2005): o coeficiente de Akaike também penaliza o número de parâmetros, uma vez que seu aumento tende a fazer com que o modelo descreva erros aleatórios ou ruídos, ao invés dos relacionamentos entre as variáveis em estudo (overfitting). A escolha entre modelos candidatos estaria entre aqueles que apresentassem os menores valores.

\section{Resultados e discussão}

\section{Qualidade do posicionamento GNSS}

Apenas a RN 2038U apresentou erro padrão superior a $40 \mathrm{~mm}( \pm 56$ $\mathrm{mm}$ ) sendo que a média, mediana e moda dos erros padrões das alturas elipsoidais $\left(h_{G N S S}\right)$ das RNs de seu grupo não difeririam caso a RN fosse suprimida do conjunto (Tabela 1). Por essa razão, a mesma foi mantida no conjunto dos dados para ajuste dos modelos. 
O erro padrão médio absoluto das RNs do grupo “20xxx” foi de aproximadamente $65 \%$ do erro padrão médio absoluto das RNs do grupo "14xxx", mas com uma variação maior (desvio-padrão de 14 mm e 8 mm, respectivamente). O erro padrão absoluto máximo do grupo "20xxx" chegou a $56 \mathrm{~mm}$ em razão da inclusão da RN 2038U, senão seria de 33 mm, semelhante ao grupo "14xxx".

Os valores alcançados indicam que o posicionamento por GNSS nas RNs da RAAP são aceitáveis, tanto para calibração do modelo como para sua validação.

Tabela 1 - Estatísticas descritivas dos erros padrões absolutos das alturas elipsoidais $\left(h_{\text {GNSS }}\right)$ das RNs da RAAP determinadas por posicionamento GNSS

\begin{tabular}{|c|c|c|c|}
\hline \multirow{2}{*}{ Estatística } & \multicolumn{2}{|c|}{ RRNN “20xxx" } & \multirow{2}{*}{ RRNN “14xxx" } \\
\cline { 2 - 3 } & Com 2038U & Sem 2038U & \\
\hline Média (m) & 0,016 & 0,014 & 0,023 \\
\hline Mediana (m) & 0,011 & 0,011 & 0,003 \\
\hline Moda (m) & 0,008 & 0,008 & 0,018 \\
\hline Desvio padrão (m) & 0,014 & 0,010 & 0,008 \\
\hline Curtose & 2,951 & $-0,841$ & $-2,695$ \\
\hline Assimetria & 1,528 & 0,543 & 0,326 \\
\hline Amplitude (m) & 0,054 & 0,031 & 0,016 \\
\hline Mínimo (m) & 0,002 & 0,002 & 0,015 \\
\hline Máximo (m) & 0,056 & 0,033 & 0,031 \\
\hline Shapiro-Wilk & 0,861 & & 0,764 \\
& $(\mathrm{p}$-valor $=0,015)$ & & $(\mathrm{p}$-valor $=0,018)$ \\
\hline
\end{tabular}

Correlação entre as variáveis $E_{\text {GNSS' }} N_{\text {GNSS' }} h_{\text {GNSS }}$

As correlações entre as variáveis ou quantidades utilizadas para calibração do modelo foram avaliadas pela matriz de correlação (Tabela 2).

Tabela 2 - Matriz de correlações entre as variáveis $\mathrm{E}_{\text {GNSS }} \mathrm{N}_{\text {GNSS'}} \mathrm{h}_{\text {GNSS }}$ das RNs do grupo "20xxx" utilizadas como variáveis independentes na calibração do modelo

\begin{tabular}{|c|c|c|c|c|}
\hline \multirow{2}{*}{ Coeficiente } & \multirow{2}{*}{ Variável } & $\mathbf{a}_{2}$ & $\mathbf{a}_{3}$ & $\mathbf{a}_{\mathbf{4}}$ \\
\cline { 3 - 5 } & & $\mathbf{E}_{\text {GNSS }}$ & $\mathbf{N}_{\text {GNSS }}$ & $\mathbf{h}_{\text {GNSS }}$ \\
\hline $\mathrm{a}_{2}$ & $\mathrm{E}_{\text {GNSS }}$ & 1 & & \\
\hline $\mathrm{a}_{3}$ & $\mathrm{~N}_{\text {GNSS }}$ & 0,024 & 1 & \\
\hline $\mathrm{a}_{4}$ & $\mathrm{~h}_{\text {GNSS }}$ & $-0,514$ & $-0,259$ & 1 \\
\hline
\end{tabular}


As variáveis $E$ e $N$ apresentaram correlação linear baixa, enquanto que essas variáveis apresentaram alguma correlação linear negativa com a variável $h$, o que indica que $h$ tende a aumentar nas direções norte e leste na região de estudo.

\section{Teste de significância dos coeficientes do modelo}

Os coeficientes do modelo foram obtidos por regressão linear múltipla (Tabela 3) e seus valores analisados estatisticamente (ANOVA). O teste $\mathrm{F}(\alpha=0,05)$ indicou a rejeição da hipótese de nulidade destes coeficientes, ou seja, pelo menos um deles se mostrou significativo.

Tabela 3 - Estatísticas para análise da significância dos coeficientes do modelo obtidos por regressão linear múltipla *

\begin{tabular}{|c|c|c|c|c|c|c|c|}
\hline \multicolumn{2}{|c|}{ Coeficiente } & Valor & Erro padrão & Estatística & valor-p & $95 \%$ & $95 \%$ \\
\hline$a_{1}$ & Interseção & $-93,84164326$ & 7,948 & $-11,81$ & $\underline{2,54 \times 10^{0.08}}$ & $-111,013$ & $\underline{-76,671}$ \\
\hline$a_{2}$ & $E_{\text {GNSS }}$ & $\underline{9,38952 \times 10^{06}}$ & $1,04 \times 10^{06}$ & $\underline{9,02}$ & $\underline{5,90 \times 10^{-007}}$ & $\underline{7,14 \times 10^{06}}$ & $1,16 \times 10^{05}$ \\
\hline$a_{3}$ & $\mathrm{~N}_{\text {GNSS }}$ & $\underline{1,19251 \times 10^{05}}$ & $1,12 \times 10^{06}$ & $\underline{10,67}$ & $\underline{8,45 \times 10^{-0.08}}$ & $\underline{9,51 \times 10^{06}}$ & $1,43 \times 10^{05}$ \\
\hline$a_{4}$ & $\mathrm{~h}_{\text {GNSS }}$ & 1,000124712 & $3,34 \times 10^{04}$ & $\underline{2993,88}$ & $\underline{2,43 \times 10^{-39}}$ & $\underline{0,999}$ & $\underline{1,001}$ \\
\hline
\end{tabular}

*Sublinhados com linha simples indicam aceitação, por se encontrarem dentro dos limites estatísticos, e duplos sublinhados indicam não aceitação estatística.

Os valores de todos os coeficientes do modelo se apresentaram dentro dos limiares superior e inferior de 95\% de confiança, sendo que nenhum dos intervalos respectivos incluiu a possibilidade de valor nulo. Os coeficientes provaram ser robustos ( $\mathrm{t}>2,160369$; $\mathrm{p}<0,05)$ e confiáveis para as estimativas das alturas ortométricas na região de estudo, seja na calibração, seja na validação.

Quanto aos resíduos do ajustamento (Tabela 4), o teste de ShapiroWilk indica a não rejeição da hipótese de nulidade ao nível de 5\% de significância. Portanto, os resíduos dos coeficientes obtidos na calibração seguem uma distribuição aproximadamente normal. 
Tabela 4 - Parâmetros estatísticos para avaliação dos resíduos

\begin{tabular}{|c|c|}
\hline Critério & Resíduos da Calibração \\
\hline Estatística: Shapiro-Wilk & $0,960$ ( $\mathrm{p}$-valor $=0,64)$ \\
\hline EMQ & 0,0012 \\
\hline Akaike & 0,2423 \\
\hline RQMR & 0,0298 \\
\hline $\mathrm{R}^{2}$ & 0,999999902 \\
\hline R $^{2}$ Ajustado & 0,99999879 \\
\hline Erro padrão & 0,034 \\
\hline Critério & Resíduos da Validação \\
\hline EM0 & 0,0015 \\
\hline Akaike & 0,3172 \\
\hline RQMR & 0,0341 \\
\hline
\end{tabular}

O EMQ apresentou-se em torno de $1 \mathrm{~mm}$ e o RQMR inferiores a $30 \mathrm{~mm}$ na calibração, enquanto que na validação tenderam a aumentar. Ambos ficaram abaixo do limite de aceitação do erro padrão de $40 \mathrm{~mm}$ estabelecido para $\mathrm{h}_{\mathrm{GNSS}}$. De acordo com os parâmetros $\mathrm{R}^{2}$ e $\mathrm{R}^{2}$ Ajustado, o modelo apresenta bons índices de explicação da variável H em função das variáveis $\mathrm{E}, \mathrm{N}$ e h.

Conclui-se que o modelo se apresenta consistente, tanto do ponto de vista da calibração dos seus parâmetros, como da capacidade de estimativa de $\mathrm{H}$ nos locais não amostrados. Portanto, a aplicação do modelo para estimativa de $\mathrm{H}$ se justifica pela consistência dos coeficientes calibrados e pela qualidade das estimativas. Contudo, observa-se que sua validade se restringe a área de estudo, especificamente à bacia hidrográfica do rio Carahá, podendo se estender até a fronteira sul do município de Lages.

O fato de este modelo incluir as coordenadas projetivas UTM, bem como estimar diretamente a altura ortométrica $(\mathrm{H})$, podem ser considerados como aspectos positivos para sua utilização. No entanto, observa-se que a coordenada UTM E é dependente da zona ou fuso UTM e seu referencial muda conforme o meridiano central do mesmo. Por essa razão, o método aqui apresentado ficaria limitado a regiões onde não há transição de fuso.

\section{Aplicação do modelo}

O modelo foi aplicado para avaliar a qualidade altimétrica da base cartográfica da cidade de Lages, obtida por restituição fotogramétrica em 
2003, pertencentes à Prefeitura Municipal (PML) e publicada na escala 1:2000, de acordo com o PEC Classe A.

A análise da qualidade altimétrica de um mapa depende da equidistância das curvas de nível (Brasil, 1984) e da qualidade com que se obtêm as alturas dos pontos utilizados como amostras para validação no terreno. Nos mapas analógicos, as alturas são impressas junto aos pontos cotados; nos mapas digitais, oriundos de restituição aerofotogramétrica, as alturas desses pontos usualmente são registradas numericamente junto aos mesmos. Presume-se, portanto, que seus valores sejam equivalentes aos valores esperados quando testados no terreno.

A análise foi realizada sobre duas amostras de pontos cotados, geradas por escolha aleatória do conjunto total de 11.587 pontos, utilizando a ferramenta Create Random Points do aplicativo ArcGis 10. Estabeleceu-se o critério de uma distância média de $400 \mathrm{~m}$ entre os pontos, resultando, na amostra 1, 86 pontos, não localizados em intervias e, na amostra 2, 71 pontos localizados nas intervias (Figura 5). Em virtude dos pontos da amostra 1 poderem se situar em locais de difícil acesso, como interior de propriedades, optou-se por selecionar um ou dois pontos nas proximidades, de forma a garantir a amostragem naquela região.

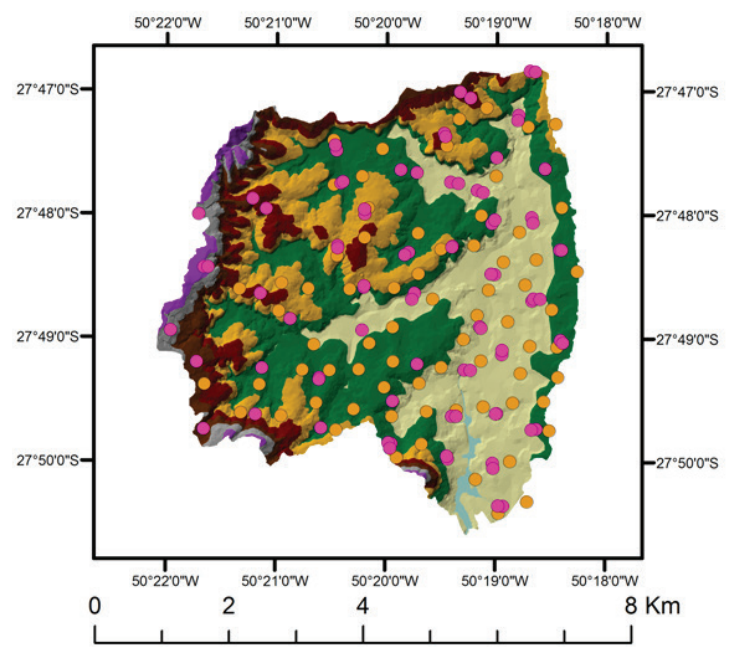

Legenda

- Amostra 1

- Amostra 2 - Intervias

\section{Elevação (m)}

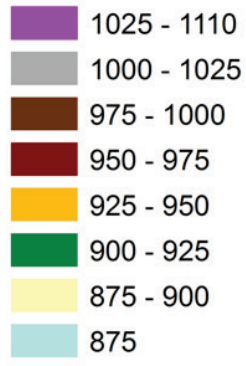

Figura 5- Distribuição dos conjuntos de pontos amostrais 1 e 2 para avaliação da qualidade altimétrica da base cartográfica da PML 
Os pontos foram ocupados a campo e posicionados por GNSS com os mesmos receptores do posicionamento das RNs da RAAP. As coordenadas projetivas UTM destes pontos foram extraídas da base cartográfica. O marco M6 (Quadro1) localizado no campus da UDESC em Lages foi utilizado como base. Estabeleceu-se um erro padrão máximo tolerável de $40 \mathrm{~mm}$ no desvio horizontal e vertical.

Tabela 5 - Coordenadas do marco M6 no sistema SIRGAS 2000 utilizado como base

\begin{tabular}{|c|c|c|}
\hline Coordenadas elipsoidais & Coordenadas planas UTM (m) & Precisões (m) \\
\hline Latitude $(\varphi)=27^{\circ} 47^{\prime} 33,74209^{\prime \prime} S$ & $\mathrm{~N}=6925567,555$ & $\Delta \mathrm{N}=0,003$ \\
\hline $\begin{array}{c}\text { Longitude }(\lambda)=50^{\circ} 18^{\prime} \\
23,76927^{\prime \prime} W\end{array}$ & $\mathrm{E}=568307,554$ & $\Delta \mathrm{E}=0,003$ \\
\hline Altura elipsoidal $(\mathrm{h})=929,331 \mathrm{~m}$ & $(\mathrm{~h})=929,331$ & $\Delta \mathrm{h}=0,008$ \\
\hline
\end{tabular}

Os resíduos dos $i$-ésimos pontos amostrais $\left(\mathrm{v}_{\mathrm{i}}\right)$ ou diferenças entre os valores observados $\left(\mathrm{V}_{\mathrm{obs}}=\mathrm{H}_{\mathrm{PML}}\right)$ e esperados $\left(\mathrm{V}_{\text {esp }}=\mathrm{H}_{\text {MODELO }}\right.$ ) foram calculados de acordo com a equação 10, cujas estatísticas descritivas estão na Tabela 5 .

$$
v_{i}=H_{P M L}-H_{M O D E L O}
$$

Tabela 6 - Estatísticas dos resíduos nos pontos amostrais

\begin{tabular}{|c|c|c|}
\hline Estatística & Amostra 1 & Amostra 2 \\
\hline Média $(\mathrm{m})$ & $-0,447$ & $-0,668$ \\
\hline Erro padrão $(\mathrm{m})$ & 0,041 & 0,079 \\
\hline Mediana $(\mathrm{m})$ & $-0,470$ & $-0,604$ \\
\hline Desvio padrão $(\mathrm{m})$ & $\pm 0,383$ & $\pm 0,666$ \\
\hline Curtose & 0,136 & 0,787 \\
\hline Assimetria & 0,134 & $-0,719$ \\
\hline Amplitude (m) & 2,012 & 3,396 \\
\hline Mínimo (m) & $-1,433$ & $-2,895$ \\
\hline Máximo (m) & 0,579 & 0,501 \\
\hline $\mathrm{n}$ & 86 & 71 \\
\hline Nível de confiança (95.0\%) & 0,082 & 0,158 \\
\hline Teste de Normalidade & & 0,966 \\
\hline Shapiro-Wilk & 0,966 & 0,052 \\
\hline p-valor & 0,052 & \\
\hline
\end{tabular}


$\mathrm{Na}$ amostra 1, 83\% dos resíduos situaram-se entre 0,0 m e -1,0 m; na amostra 2, esse percentual foi de 62\% (Figura 6). Apenas 10,5\% dos resíduos foram positivos na amostra 1 e 14,1\% na amostra 2, sendo ambos com resíduos de até $+0,6 \mathrm{~m}$. Apenas um ponto da amostra 2 apresentou resíduo na classe de $-3,0 \mathrm{~m}$ a $-2,5 \mathrm{~m}$ e um na classe $-2,5 \mathrm{~m}$ a $-2,0 \mathrm{~m}$, representando cada um 1,4\% dos pontos amostrados.

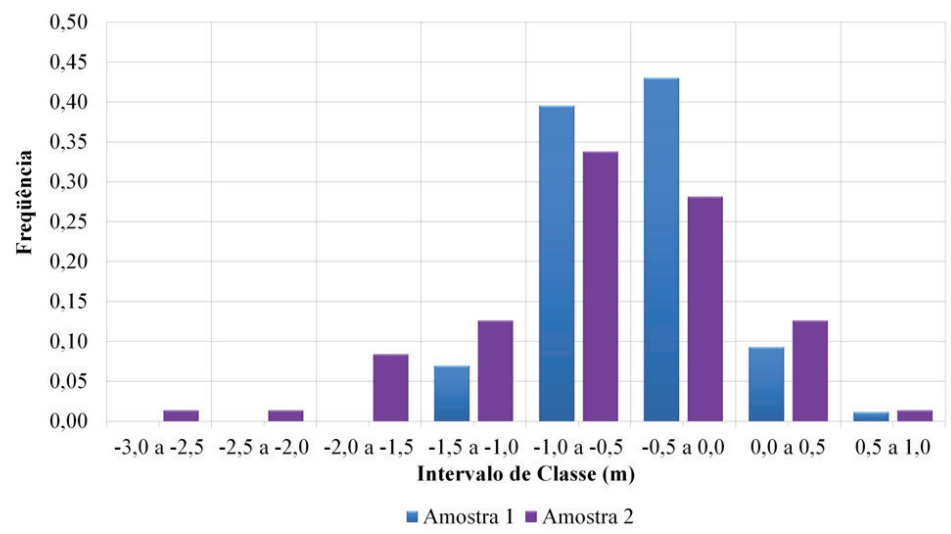

Figura 6 - Histograma dos resíduos das amostras 1 e 2

Esses dados sugerem que de 10 a 14\% dos pontos da base cartográfica podem estar superestimando a altura ortométrica esperada em até $+0,6 \mathrm{~m}$ e $2,8 \%$ de $-3,0 \mathrm{~m}$ a $-2,5 \mathrm{~m}$. Por outro lado, de 60 a $80 \%$ dos pontos desta base podem estar apresentando desvios da ordem de até -1,0 m.

Quando os resíduos são comparados com os limites estabelecidos pelo Decreto n $^{\circ} 89.817$ para a PEC de mapas classe A, observa-se que, para a altimetria, o erro-padrão limite de $1 / 3$ da equidistância das curvas de nível na escala 1:2000 é da ordem de $0,3 \mathrm{~m}(1 \mathrm{~m} \times 1 / 3)$ e que, portanto, os erros-padrões tanto da amostra 1 como da amostra 2 ficaram bem abaixo deste limite (Tabela 5). O PEC desta base cartográfica corresponderia, de acordo com o referido decreto, a $0,5 \mathrm{~m}$, ou seja, $1 / 2$ da equidistância das curvas de nível ou igual a 1,6449 vezes $0,3 \mathrm{~m}$.

$\mathrm{Na}$ amostra 1 , apenas $53,5 \%$ dos pontos atenderiam ao critério de estarem entre $\pm 0,5 \mathrm{~m}$ correspondente à PEC. Na amostra 2, são 40,8\%. Portanto, de acordo com os pontos verificados a campo, a qualidade 
cartográfica dos pontos cotados indicaria o não atendimento aos parâmetros de qualidade determinados pela legislação brasileira.

Com relação à distribuição espacial dos resíduos, essa se mostrou aleatória em ambas as amostras (Figura 7). Nos locais amostrados em pontos vizinhos (amostra 1), a magnitude dos resíduos mostrou-se consistente, indicando consistência do método amostral utilizado.
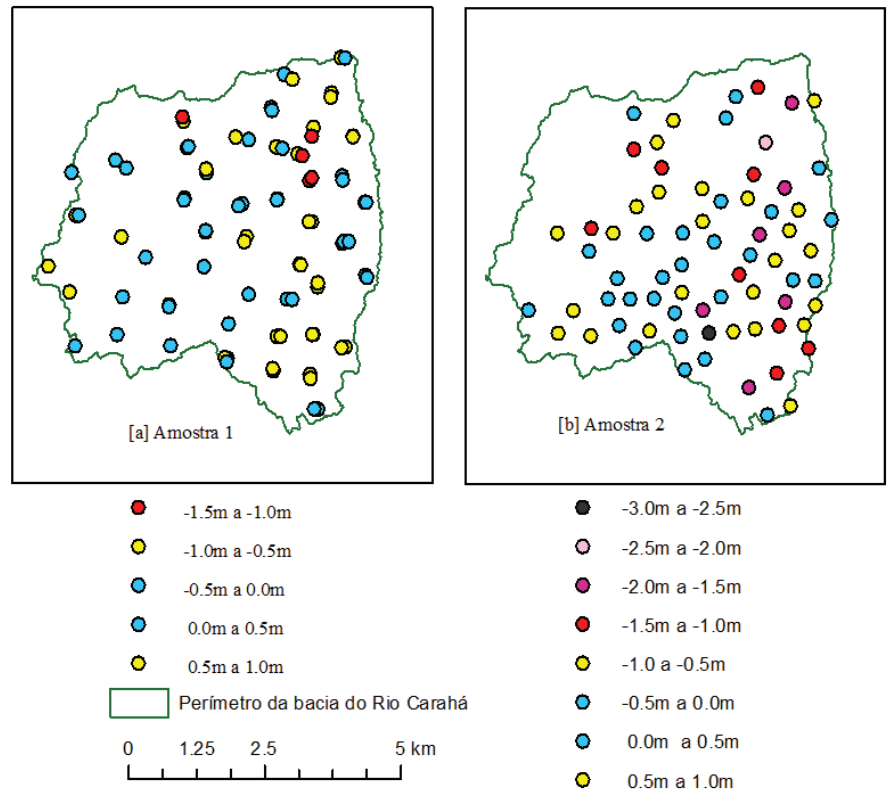

Figura 7 - Distribuição espacial dos resíduos (m) das amostras 1

e 2. Símbolos maiores indicam resíduos acima do PEC

\section{Conclusões}

Este trabalho apresentou um modelo linear local para estimativa das alturas ortométricas referenciadas à RAAP. O modelo mostrou-se válido para um campo de até $50 \mathrm{~km}$ ao sul da cidade de Lages. O modelo indicou que de $40 \%$ a $53 \%$ dos pontos cotados da base cartográfica da cidade atenderiam ao critério de estarem entre $\pm 0,5 \mathrm{~m}$ correspondente à PEC classe A. 
Nota

1 - A aproximação nesta equação é devido à desconsideração do desvio da vertical que, para fins práticos, pode ser negligenciado (Genro, 2006).

\section{Referências}

ABDULLAH, K. A. Improving geoidal height estimates from global geopotential model using regression model and GPS data. Bulelin Geoinformasi, v.1, n. 3, p.112 -118, 1997.

ARANA, J. M. O uso do GPS na elaboração de carta geoidal. Tese (Doutorado em Ciências) - Universidade Federal do Paraná, Curitiba, 2000. 180 f.

BRAGA, L. P. V. Introdução à mineração de dados. Rio de Janeiro: E-Papers Serviços Editoriais, 2005. 212 p.

BRASIL. Decreto-lei no 89.817, de 20 de junho de 1984. Instruções Reguladoras das Normas Técnicas da Cartografia Nacional. Diário Oficial da União, Brasília, 1984. Disponível em: <http://www.planalto.gov.br/ccivil_03/decreto/1980-1989/ D89817.htm>. Acesso em: 10 ago. 2015.

FEATHERSTONE, W. E.; DENTITH, M. C.; KIRBY, J. F. Strategies for the accurate determination of orthometric heights from GPS. Survey Review, v. 34, n. 267, p. 278-296, 1998.

FOTOPOULOS, G. An analysis on the optimal combination of geoid, orthometric and ellipsoidal height data. Thesis (Doctor of Philosophy) - University of Calgary, Calgary, 2003. $258 \mathrm{f}$.

GENRO, R. S. Determinação de um geóide gravimétrico para o Estado do Paraná utilizando colocação por mínimos quadrados. Dissertação (Mestrado em Ciências Geodésicas) - Universidade Federal do Paraná, Curitiba, 2006. 136 f.

IBGE (Instituto Brasileiro de Geografia e Estatística). Ajustamento simultâneo da rede altimétrica de alta precisão do sistema geodésico brasileiro. Relatório Técnico, 2011. $60 \mathrm{p}$.

. RPMG - Rede maregráfica permanente para geodésia. Disponível em $<\mathrm{http} / / / \mathrm{w} w \mathrm{w}$.ibge.gov.br/home/geociencias/geodesia/rmpg/default_rmpg_int. shtm?c=10>. Acesso em: 27 out. 2013.

KHALID, A. B.; SOM, Z. A. The modelling of corrective surface for GPS height conversion in klang valley. Geoinformation Science Journal, vol. 11, n. 1, pp: 3350, 2011.

MATOS, A. C. O. C; BLITZKOW, D.; GUIMARÃES, G. N.; LOBIANCO, M. C. B.; COSTA, S. M. A. Validação do MAPGEO2010 e comparação com modelos do geopotencial recentes. Boletim de Ciências Geodésicas, v. 18, n. 1, p.101-122, 2012. 
MONTGOMERY, C.; RUNGER, G.C. Estatística aplicada e probabilidade para engenheiros. Rio de Janeiro: LTC, 2009. 496 p.

PAVLIS, N. K.; HOLMES, S. A.; KENYON, S. C.; Factor, J. K. The development and evaluation of the Earth Gravitational Model 2008 (EGM2008). Journal of Geophysical Research: Solid Earth, v. 117, Issue B4, 2012.

ZHONG, D. Robust estimation and optimal selection of polynomial parameters for the interpolation of GPS geoid heights. Journal of Geodesy, v. 71, n. 9, p. 552$561,1997$.

Sílvio Luís Rafaeli Neto - Possui Graduação em Agronomia pela Universidade do Estado de Santa Catarina. Mestrado em Ciências Geodésicas pela Universidade Federal do Paraná e Doutorado em Engenharia pela Escola Politécnica da Universidade de São Paulo. Atualmente é professor associado da Universidade do Estado de Santa Catarina.

Leonardo Josoé Biffi - Possui Graduação em Agronomia pela Universidade do Estado de Santa Catarina. Mestrado em Produção Vegetal pela Universidade do Estado de Santa Catarina. Atualmente é professor assistente da Universidade do Estado de Santa Catarina.

Recebido para publicação em 6 de outubro de 2015 Aceito para publicação em 17 de Novembro de 2015 